\title{
Leishmania braziliensis replication protein A subunit 1: molecular modelling, protein expression and analysis of its affinity for both DNA and RNA
}

\author{
Paola A Nocua', Cesar A Ramirez', George E Barreto², Janneth González², José M Requena ${ }^{3}$ \\ and Concepción J Puerta ${ }^{1 *}$
}

\begin{abstract}
Background: Replication factor A (RPA) is a single-strand DNA binding protein involved in DNA replication, recombination and repair processes. It is composed by the subunits RPA-1, RPA-2 and RPA-3; the major DNA-binding activity resides in the subunit 1 of the heterotrimeric RPA complex. In yeast and higher eukaryotes, besides the three basic structural DNA-binding domains, the RPA-1 subunit contains an N-terminal region involved in protein-protein interactions with a fourth DNA-binding domain. Remarkably, the N-terminal extension is absent in the RPA-1 of the pathogenic protozoan Leishmania (Leishmania) amazonensis; however, the protein maintains its ability to bind ssDNA. In a recent work, we identify Leishmania (Viannia) braziliensis RPA-1 by its specific binding to the untranslated regions of the HSP70 mRNAs, suggesting that this protein might be also an RNA-binding protein.
\end{abstract}

Methods: Both rLbRPA-1 purified by His-tag affinity chromatography as well as the in vitro transcribed L. braziliensis 3' HSP70-II UTR were used to perform pull down assays to asses nucleic acid binding properties. Also, homology modeling was carried out to construct the LbRPA-1 tridimensional structure to search relevant amino acid residues to bind nucleic acids.

Results: In this work, after obtaining the recombinant L. braziliensis RPA-1 protein under native conditions, competitive and non-competitive pull-down assays confirmed the single-stranded DNA binding activity of this protein and demonstrated its interaction with the 3' UTR from the HSP70-II mRNA. As expected, this protein exhibits a high affinity for ssDNA, but we have found that RPA-1 interacts also with RNA. Additionally, we carried out a structural analysis of L. braziliensis RPA-1 protein using the X-ray diffraction structure of Ustilago maydis homologous protein as a template. Our results indicate that, in spite of the evolutionary divergence between both organisms, the structure of these two RPA-1 proteins seems to be highly conserved.

Conclusion: The LbRPA-1 protein is a ssDNA binding protein, but also it shows affinity in vitro for the HSP70 mRNA; this finding supports a possible in vivo role in the HSP70 mRNA metabolism. On the other hand, the three dimensional model of Leishmania RPA-1 serves as a starting point for both functional analysis and its exploration as a chemotherapeutic target to combat leishmaniasis.

Keywords: Replication protein A (RPA), RPA subunit 1, Single-stranded DNA binding protein, RNA binding protein, Leishmania braziliensis

\footnotetext{
* Correspondence: cpuerta@javeriana.edu.co

'Laboratorio de Parasitología Molecular, Facultad de Ciencias, Pontificia Universidad Javeriana, Carrera 7 No 43-82, Edificio 50, Laboratorio 113, Bogotá, Colombia

Full list of author information is available at the end of the article
} 


\section{Background}

The leishmaniasis, caused by protozoan parasites belonging to the genus Leishmania, affects the population of 98 countries on the 5 continents, being 350 million people at risk of infection, 12 million are currently infected and each year 2 million new cases occur [1]. In the Americas, Leishmania (Viannia) braziliensis is the major agent of mucocutaneous leishmaniasis (LMC) and one of the six causative species of cutaneous leishmaniasis (LC) [2]. As there are no vaccines against any type of leishmaniasis and the treatment options are limited, efforts for developing effective vaccines and drugs should be done urgently.

Replication protein A (RPA) is the main eukaryote single-stranded DNA (ssDNA) binding protein, being essential for DNA replication, recombination and repair processes [3]. It has also been involved in cell cycle and DNA damage checkpoint activation $[3,4]$. In mammals, this protein, composed by the subunits RPA-1 (70 kDa), RPA-2 (32-34 kDa) and RPA-3 (14 kDa), plays two functional roles. On the one hand, the protein maintains ssDNA in an extended structure and protects solventexposed DNA bases from undesired chemical modifications. On the other hand, RPA interacts with several proteins in order to orchestrate different cellular processes regarding DNA maintenance [5].

The RPA heterotrimer consists of six ssDNA binding domains (DBD), also known as OB (oligonucleotide/ oligosaccharide-binding) fold, each one consisting of five $\beta$-strands arranged in a $\beta$-barrel [6]. The major DNAbinding activity is found in the subunit 1 of the RPA protein (RPA-1), which is also responsible for interaction with replication and repair proteins. It exhibits a modular structure having four out of the six RPA DBDs existing in the heterotrimeric RPA. These domains, arranged in tandem, are denoted as DBD-F, DBD-A, DBD-B, and DBD-C $[7,8]$. The $\mathrm{N}$-terminal region (RPA1N), besides bearing the DBD-F domain, is involved in interactions with other DNA metabolism proteins $[9,10]$. Indeed, the initiation of the DNA damage response by RPA is mediated by the RPA-1 subunit through protein-protein interactions involving its $\mathrm{N}$-terminal domain [11].

A homologue of RPA was biochemically purified from the trypanosomatid Crithidia fasciculata by Brown and co-workers [12]. The purified complex was found to consist of three polypeptides of 51,28 , and $14 \mathrm{kDa}$ that binds single-stranded DNA via the large subunit; the complex was localized within the nucleus. In a subsequent work, the genes encoding the 51-kilodalton subunit (p51) and the 28-kilodalton subunit (p28) of RPA were cloned and sequenced [13]. The predicted p51 polypeptide has sequence similarity to the corresponding subunits from humans and Saccharomyces cerevisiae, but it is smaller, lacking of a segment of approximately
$20 \mathrm{kDa}$ in the N-terminal region. In Leishmania amazonensis, a species belonging to the subgenera Leishmania and associated with different clinical manifestations such as cutaneous (CL), mucocutaneous (MCL) and visceral (VL) leishmaniasis [14], the subunit 1 of replication protein A (RPA-1) was identified by its association with Grich telomeric sequences [15]. Of note, this protein also showed affinity for RNA oligonucleotides containing the cognate telomeric sequence [15]. After sequencing of the gene and deducing of the encoded amino acid sequence, it was found that LaRPA-1, like the CfRPA-1 (see above), lacked the N-terminal RPA70N domain (present in human and yeast RPA-1), but shared with hRPA-1 and yRPA-1 a canonical N-terminal tRNA_anti domain, which is an $\mathrm{OB}$ (oligonucleotide/oligosaccharide-binding) fold structure [16]. More recently, these authors found evidence that the natural absence of RPA1N domain in LaRPA-1 does not impair its participation in DNA damage response and telomere protection [17].

In a recent work, we identified the $L$. braziliensis RPA-1 (LbRPA-1) protein based on its binding to the $5^{\prime}$ and 3' untranslated regions (UTR) of the $70 \mathrm{kDa}$ heat shock protein (HSP70) mRNAs [18]. In order to further analyze the role played by LbRPA-1, as a first step, we conducted a structural analysis and its tertiary structure was modelled based on the X-ray diffraction structure of Ustilago maydis RPA-1 protein, which has been solved recently [19]. In spite of the sequence divergence existing between both proteins, L. braziliensis RPA-1 protein conforms the typical DNA-binding domains of RPA proteins. Additionally, we expressed the LbRPA-1 protein in Escherichia coli and the purified recombinant protein was used to study its affinity for both DNA and RNA. As expected, the rLbRPA-1 binds ssDNA with high affinity, but remarkably it also binds RNA, suggesting a possible role for this protein in mRNA metabolism.

\section{Methods}

\section{Parasite cultures and nucleic acid extraction}

Promastigotes of L. braziliensis MHOM/BR/75/M2904 were cultured in vitro at $26^{\circ} \mathrm{C}$ in Schneider's insect medium (Sigma Aldrich, Inc., St. Louis, MO, USA) supplemented with $20 \%$ heat-inactivated fetal calf serum (Eurobio, Inc., Les Ulis, France), and $0.1 \mu \mathrm{g} / \mathrm{mL}$ of 6 biopterin (Sigma Aldrich, Inc., St. Louis, MO, USA). Total DNA from parasite cells was isolated using the phenol-chloroform-isoamilic alcohol method [20].

\section{Cloning and sequence analysis of the $L$. braziliensis RPA-1 gene}

The L. braziliensis RPA-1 (LbRPA-1) coding region was amplified from genomic DNA by PCR using the primers RPA1F (5'-GGATCCATCGTGATGCAGCAG CCG-3') and RPA1R (5'-CTGCAGTCACACGTACGC 
CTCGATGAG-3'). These primers were designed from the LbrM.28.1990 entry existing in the L. braziliensis GeneDB database (http://genedb.org). These primers amplify a fragment of $1422 \mathrm{bp}$ and contain the BamHI and PstI restriction sites (italics letters in the sequence), respectively. The PCR mix reaction (final volume of $20 \mu \mathrm{L}$ ) included: $1 \times$ reaction buffer $(10 \mathrm{mM}$ Tris- $\mathrm{HCl} \mathrm{pH}$ 9.0, 50 $\mathrm{mM} \mathrm{KCl}, 0.1 \%$ Triton X-100), $2 \mathrm{mM} \mathrm{MgCl}_{2}, 0.4 \mathrm{mM}$ of dNTP mix, $0.5 \mu \mathrm{M}$ of each primer, 0.06 units per $\mu \mathrm{L}$ of expand high fidelity enzyme (Roche, Inc., Mannheim, Germany) and $3.3 \mathrm{ng} / \mu \mathrm{L}$ of L. braziliensis total DNA. An MJ Research PTC-100 DNA thermocycler was used for the reaction with the following amplification profile: $95^{\circ} \mathrm{C} / 3$ min, 3 cycles at $92^{\circ} \mathrm{C} / 30 \mathrm{~s}, 56^{\circ} \mathrm{C} / 30 \mathrm{~s}, 72^{\circ} \mathrm{C} / 1.5 \mathrm{~min}$, followed by 36 cycles at $92^{\circ} \mathrm{C} / 30 \mathrm{~s}, 64^{\circ} \mathrm{C} / 30 \mathrm{~s}, 72^{\circ} \mathrm{C} / 1.5 \mathrm{~min}$, and a final extension at $72^{\circ} \mathrm{C}$ for $10 \mathrm{~min}$ and $12^{\circ} \mathrm{C}$ as PCR stop temperature. The amplified fragment was resolved in agarose gels and visualized under UV exposure after ethidium bromide staining. PCR product was excised from gel, purified using a Zymoclean ${ }^{\mathrm{TM}}$ Gel DNA Recovery Kit (Zymo Research, Irvine, CA, USA) and cloned into the pGEM-T Easy plasmid (Promega, Inc., Fitchburg, WI, USA). The plasmid insert of two clones, named RPA-1A and RPA-1B, was sequenced using the Big Dye Terminators v3.1 kit (Applied Biosystem, Foster City, CA, USA) by automatic sequencing at the Servicio de Genómica (Parque Científico de Madrid, Universidad Autónoma de Madrid). The sequence of both clones was identical to the entry LbrM.28.1990.

The homology analyses of amino acids sequences of RPA-1 was carried out using the T-Coffee tool (http:// www.ebi.ac.uk/Tools/msa/tcoffee/) [21]. The domains and potential phosphorylation sites were identified by the InterProScan tool (http://www.ebi.ac.uk/Tools/pfa/iprscan/) [22,23] and the NetPhos server (http://cbs.dtu.dk/ services/NetPhos/) [24], respectively.

\section{Cloning and expression of the $L$. braziliensis RPA-1 gene}

The LbRPA1 coding region was subcloned into the pQE30 expression plasmid (QIAGEN, Inc., Hilden, Germany), and thermo competent $E$. coli cells (M15 strain) were transformed with the corresponding plasmid. The cells were grown in $200 \mathrm{~mL}$ of LB medium supplemented with $75 \mu \mathrm{g} / \mathrm{mL}$ of ampicillin and $25 \mu \mathrm{g} / \mathrm{mL}$ of kanamycin overnight at $37^{\circ} \mathrm{C}$. When the culture reached an $\mathrm{A}_{600}$ of 0.6 , protein expression was induced with $1 \mathrm{mM}$ isopropyl thio- $\beta$-galactopyranoside (IPTG); bacteria were incubated at $37^{\circ} \mathrm{C}$ for an additional $4 \mathrm{~h}$. The cells were harvested (15 min, $4696 \mathrm{~g}$ at $4^{\circ} \mathrm{C}$ ) and the pellet was suspended in $10 \mathrm{~mL}$ of lysis buffer $\left(300 \mathrm{mM} \mathrm{NaCl}, 50 \mathrm{mM} \mathrm{NaH}{ }_{2} \mathrm{PO}_{4}\right.$, $0.5 \mathrm{mg} / \mathrm{mL}$ lysozyme, $10 \mu \mathrm{g} / \mathrm{mL}$ DNase I, $10 \mu \mathrm{g} / \mathrm{mL}$ RNase A and $1 \%$ Triton $\mathrm{X}-100, \mathrm{pH} 8.0$ ), incubated on ice for $30 \mathrm{~min}$ and then $1 \mathrm{mM}$ PMSF was added. This mixture was centrifuged, and the pellet was suspended in $10 \mathrm{~mL}$ of
Urea lysis buffer A (100 mM NaH${ }_{2} \mathrm{PO}_{4}, 10$ mM Tris- $\mathrm{HCl}$ and $8 \mathrm{M}$ Urea, $\mathrm{pH} 8.0$ ), and disrupted by sonication on ice followed by centrifugation. The presence of the protein in the supernatant was confirmed by SDS-PAGE.

Protein purification was carried out through affinity chromatography using a $\mathrm{Ni}^{2+}$-NTA-Agarose resin (QIAGEN, Inc., Hilden, Germany), and following the method described by Lira and co-workers [25], with a few modifications. In brief, after binding to the nickelcolumn, the protein was refolded passing a urea gradient (from $6 \mathrm{M}$ to $1 \mathrm{M}$ ) in a solution containing $50 \mu \mathrm{g} / \mathrm{mL}$ heparin, $300 \mathrm{mM} \mathrm{NaCl}, 100 \mathrm{mM} \mathrm{NaH}_{2} \mathrm{PO}_{4}$ and $20 \%$ glycerol. Subsequently the protein was eluted in different fractions with a buffer containing $50 \mathrm{mM} \mathrm{NaH} \mathrm{PO}_{4}$, $300 \mathrm{mM} \mathrm{NaCl}, 400 \mathrm{mM}$ imidazole, $10 \mathrm{mM}$ HEPES, $50 \mu \mathrm{g} / \mathrm{ml}$ heparin, $5 \%$ glycerol and $50 \mathrm{mM}$ glycine, $\mathrm{pH}$ 7.5. The concentration of refolded protein was determined using the Micro $\mathrm{BCA}^{\mathrm{Tm}}$ protein assay kit (Thermo Scientific, Inc., Waltham, MA, USA). Finally, the purity of the protein was checked by SDS-PAGE.

\section{Cloning and expression of the $L$. braziliensis a-tubulin gene}

For cloning the $\alpha$-tubulin ORF of L. braziliensis, two oligonucleotides were designed from the entry LbrM13 V2.0200: Lb-Tub-F, GGATCCATGC GTGAGGCTAT CTGC (BamHI site in italics letters); Lb-Tub-R, CTGCAGCTAG TACTCCTCGA CGTCCT (PstI site in italics letters). PCR reaction was carried out as indicated above, using the following amplification program: $95^{\circ} \mathrm{C}$ for $5 \mathrm{~min}, 30$ repeated cycles of $30 \mathrm{~s}$ at $95^{\circ} \mathrm{C}, 30 \mathrm{~s}$ at $58^{\circ} \mathrm{C}$, and $2 \mathrm{~min}$ at $72^{\circ} \mathrm{C}$. A final extension of $5 \mathrm{~min}$ at $72^{\circ} \mathrm{C}$ was included. The PCR product was digested with $\mathrm{BamHI}$ and Pst I and cloned into the corresponding restriction sites of plasmid pQE30 (QIAGEN, Inc., Hilden, Germany). After checking the sequence correctness, the resulting clone was used for protein expression in E. coli M15 strain. Protein expression and purification was done following identical procedures to those used for the rLbRPA-1 protein (see above).

\section{In vitro transcription and non-radioactive labeling of the L. braziliensis 3' HSP70-II UTR}

The L. braziliensis 3' HSP70-II UTR region was PCR amplified as described previously using pTLb3H70-11B clone as a template [18]. The $\mathrm{T} 7$ promoter sequence was included in the forward oligonucleotide in order to obtain a PCR product bearing at its $5^{\prime}$-end the recognition site for the T7 RNA polymerase. For in vitro transcription, the MEGAscript ${ }^{\oplus}$ T7 kit was used according to manufacturer's instructions (Ambion, Inc., Austin, TX, USA). Subsequently, the transcription products were treated with RNase free-DNase and the RNAs purified by the TRIZOL reagent method (Invitrogen, Inc., Carlsbad, CA, USA) 
according to Ramirez and coworkers [18]. The nonradioactive labeling was performed using a terminal transferase (Roche, Inc., Mannheim, Germany) following the manufacturer's instructions.

\section{Binding and competition assays of the L. braziliensis RPA-1 recombinant protein to a digoxigenin-labelled oligodeoxyribonucleotide}

The rLbRPA-1 protein $(40 \mu g)$ was incubated with $0.8 \mathrm{mg}$ of His-tag isolation \& Pull down dynabeads (Invitrogen, Inc., Carlsbad, CA, USA) in $1 \times$ binding buffer $\left(50 \mathrm{mM} \mathrm{NaH}{ }_{2} \mathrm{PO}_{4}, 300 \mathrm{mM} \mathrm{NaCl}, 50 \mu \mathrm{g} / \mathrm{ml}\right.$ heparin, $0.01 \%$ tween 20 and $5 \%$ glycerol) at room temperature for $10 \mathrm{~min}$ on continuous stirring. Unbound protein was removed through three rinses with $500 \mu \mathrm{l}$ of $1 \times$ binding buffer. Then, the rLbRPA- 1 bound to the beads was incubated with $12.5 \mathrm{ng}$ of Dig-Oligodeoxyribonucleotide (Dig-O, 5' Dig-GGCGAGCAGC GTCGGCCACG CAT CTCAACG CGAGCGTGCT 3') and either double stranded DNA (dsDNA), single stranded DNA (ssDNA), yeast tRNA (Roche, Inc., Mannheim, Germany) or none. Plasmid pQE30 DNA (QIAGEN, Inc., Hilden, Germany) was linearized with BamHI and used as dsDNA competitor; the ssDNA competitor was obtained after incubation at $95^{\circ} \mathrm{C}$ for $10 \mathrm{~min}$ of the BamHI-linearized pQE30. After incubation of the mixtures overnight at room temperature in $1 \times$ pull-down buffer (3.25 mM NaH2PO4 pH 7.4, $70 \mathrm{mM} \mathrm{NaCl}$, and $0.01 \%$ tween 20 ); the protein-nucleic acid complexes loaded in the beads were crosslinked by adding $1 \%$ formaldehyde, and afterwards the beads were

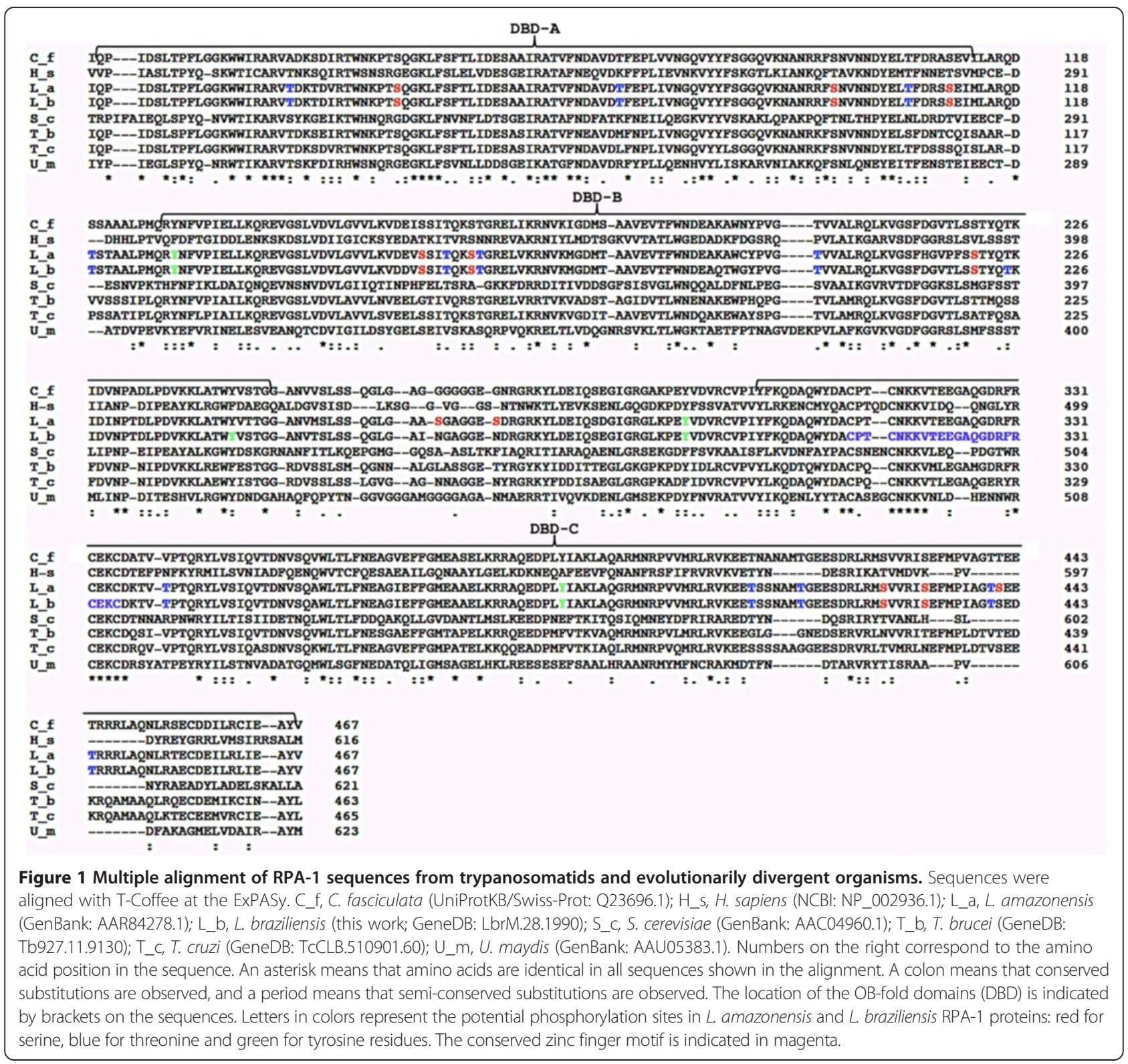


washed three times with $1 \times$ pull down buffer. Finally, the protein-nucleic acid complexes were eluted in $20 \mu \mathrm{L}$ of pull down elution buffer $(50 \mathrm{mM}$ NaH2PO4, $300 \mathrm{mM}$ $\mathrm{NaCl}, 400 \mathrm{mM}$ Imidazole, $10 \mathrm{mM}$ HEPES, $50 \mu \mathrm{g} / \mathrm{mL}$ heparin and $10 \%$ glycerol) at $90^{\circ} \mathrm{C}$ for $5 \mathrm{~min}$. The rLbRPA1nucleic acid complexes obtained were analyzed by dot immunoblotting. Immunological detection of the complexes was performed using the DIG luminescent detection kit (Roche, Inc., Mannheim, Germany). For this purpose, $2 \mu \mathrm{L}$ of the eluted samples were placed on a nylon membrane (Roche, Inc., Mannheim, Germany), fixed with UV $(1200 \mu \mathrm{J})$, washed with maleic washing buffer $(0.1 \mathrm{M}$ maleic acid, $0.15 \mathrm{M} \mathrm{NaCl} \mathrm{pH} 7.5$ and $0.3 \%$ tween 20 ), and blocked overnight on continuous stirring. Next, the membrane was incubated with antiDIG-AP conjugate (1:20000 in blocking solution) at $37^{\circ} \mathrm{C}$ during $40 \mathrm{~min}$ on continuous stirring. After extensive washing with the maleic washing buffer, the membrane was incubated in the detection buffer $(0.1 \mathrm{M}$ Tris- $\mathrm{HCl}$ and $0.1 \mathrm{M} \mathrm{NaCl} \mathrm{pH}$ 9.5); afterwards, the CSPD substrate solution was spread on membrane, which was incubated for $10 \mathrm{~min}$ at $37^{\circ} \mathrm{C}$. Finally, an x-ray film (Agfa, Inc., Mortsel, Belgium) was exposed to the membrane for $30 \mathrm{~min}$ and then developed.

Pull down assays of the $L$. braziliensis RPA- 1 recombinant protein and nucleic acids

The rLbRPA-1 protein was incubated with His-tag isolation \& Pull down dynabeads (Invitrogen, Inc., Carlsbad, CA, USA) in binding $1 \times$ buffer at room temperature for 15 min on continuous stirring. Unbound protein was removed and the protein-coupled beads were blocked by adding $5 \mu \mathrm{g}$ of recombinant L. braziliensis $\alpha$-tubulin for $20 \mathrm{~min}$. Then, the beads were incubated with single strand DNA or RNA for 20 min in pull-down $1 \times$ buffer; afterwards, the beads were washed three times with pull down buffer $0.5 \times$ and once with DEPC-treated distilled water. Finally, the protein-nucleic acid complexes were eluted in $10 \mu \mathrm{L}$ of pull down elution buffer at $90^{\circ} \mathrm{C}$ for 5 min. The rLbRPA1-nucleic acid complexes obtained were analyzed by dot immunoblotting and the signals were detected as mentioned above.

\section{Molecular modeling of the Leishmania RPA-1 proteins}

The primary sequences were analyzed by PSI-BLAST (Position-Specific Iterated-Basic Local Alignment Search, http://blast.ncbi.nlm.nih.gov/Blast.cgi?PROGRAM=blastp\& PAGE_TYPE=BlastSearch\&LINK_LOC=blasthome) tool [26] against the Protein Data Bank (PDB, http://www.rcsb. org/pdb/home/home.do) [27] in order to identify possible homologues proteins having tertiary structure resolved by experimental methods. The protein exhibiting the best BLAST/p e-value, alignment score and sequence coverage was used as template for building the 3D homology model of the L. braziliensis protein. The protein modeling was achieved using the Phyre2 server (http://www.sbg.bio.ic.ac. uk/phyre2/html/page.cgi?id=index) [28]. The predicted tertiary structures were compared with the predictions of secondary structures, and validated according to Ramachandran plot and other stereochemical parameters analyzed by the PROCHECK tool [29]. This program assesses the stereochemical quality of a given protein structure by analyzing the geometry of the residues in a given protein structure in comparison with stereochemical parameters derived from well-refined, high-resolution structures. The PROCHECK tool is available at the Swiss Model web-server (http://swissmodel.expasy.org/) [30]. Additionally, molecular modelling of the L. braziliensis RPA-1

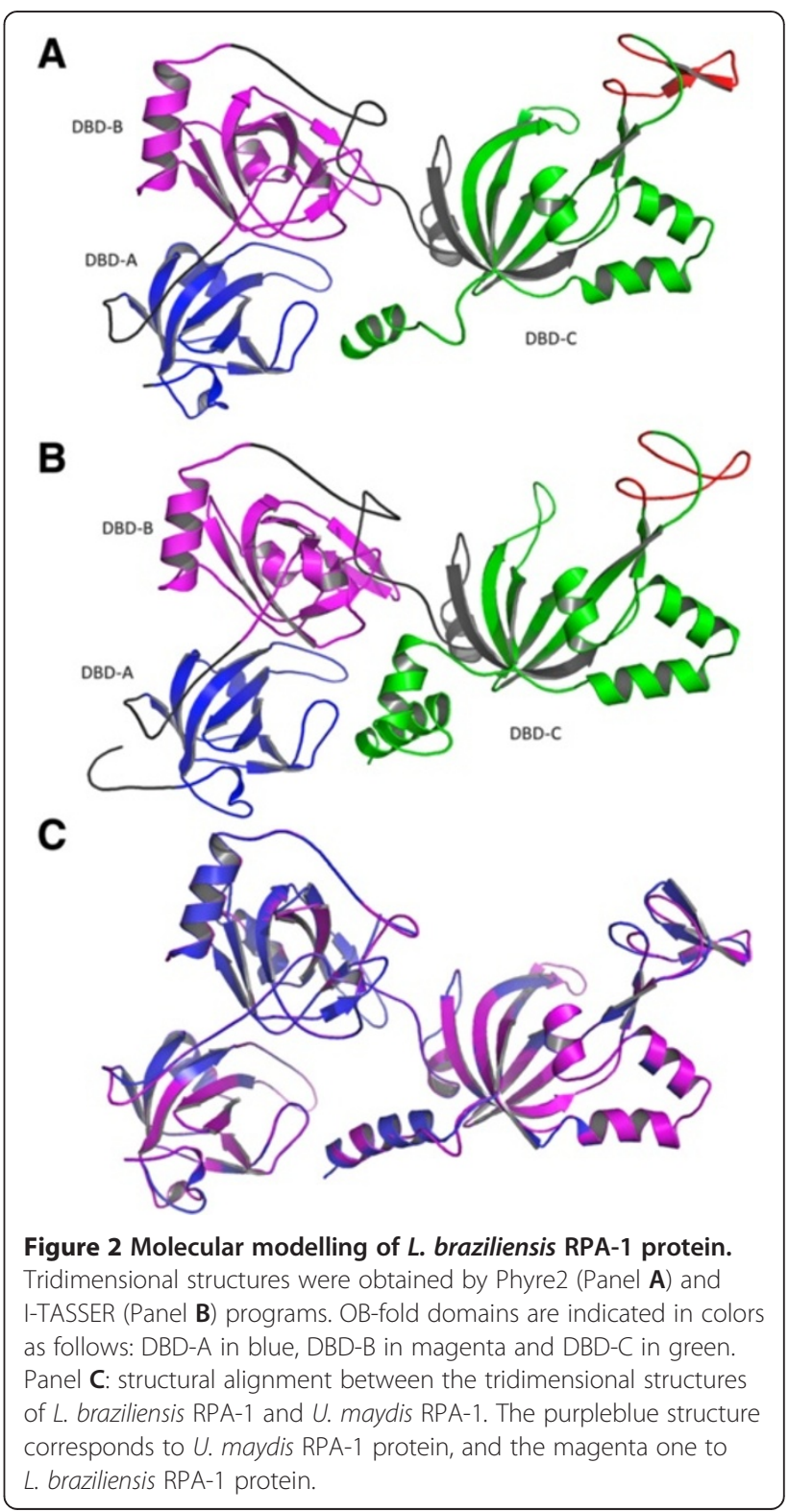


sequence was obtained using the I-TASSER server, which has been developed to generate automated full-length tridimensional protein structural predictions [31-33]. The RPA-1 protein models were visualized with the program MacPyMOL Molecular Graphics System, Version 1.5.0.4.

\section{Results and discussion}

Structural features of the $L$. braziliensis RPA-1 and determination of its tridimensional structure by molecular modeling

According to the data available at the GeneDB database, in the L. braziliensis genome exists a single-copy gene encoding RPA-1. The LbRPA-1 gene is located at chromosome 28 (LbrM.28.1990), and it is defined by an ORF encoding for a protein of 467 amino acids. The genome of Leishmania major (MHOM/IL/81/Friedlin), Leishmania infantum (JPCM5), Leishmania mexicana (MHOM//GT/ 2001/U1103), Leishmania donovani (BPK282A1), L. amazonensis (Genbank accession number AAR84278.1) and Leishmania tarentolae (Parrot-TarII) contains also a single $R P A-1$ gene, and in all species the predicted polypeptide has 467 amino acids. The alignment of L. braziliensis RPA-1 deduced amino acid sequence with homologous proteins from different organisms is shown in Figure 1. Among Leishmania species, sequence identity was higher than 95.9\%; when compared with the homologues in other trypanosomatids (Crithidia fasciculata, Trypanosoma brucei and Trypanosoma cruzi) the sequence identity remained higher than $68.8 \%$. Also significant sequence conservation (above $32.2 \%$ of sequence identity) was observed after comparison with RPA-1 proteins from evolutionarily distant organisms like Homo sapiens, Saccharomyces cerevisiae and $U$. maydis. As a remarkable feature, the RPA-1 protein in Leishmania and related trypanosomatids lacks an $\mathrm{N}$-terminal domain (RPA-1 N), present in RPA-1 from the other organisms $[13,17]$. However, the other domains of RPA-1 (i.e., DBD-A, DBD-B and DBD-C) were reliably predicted in the Leishmania proteins (Figure 1). In addition, it was found that LbRPA-1, as described for other RPA-1 proteins $[13,34,35]$, contains a C4-type zinc-finger motif, which, upon zinc coordination, stabilizes the tertiary structure of the RPA-1 C- terminal end and modulates DNA-binding [36]. The motif, $\mathrm{C}-\mathrm{X}_{2}-\mathrm{C}-\mathrm{X}_{15}-\mathrm{C}-\mathrm{X}_{2}-\mathrm{C}$, located at the $313-335$ position in L. braziliensis sequence (Figure 1), is absolutely conserved in C. fasciculata [13], but slightly different to those found in human and yeast RPA-1 subunits $\left(\mathrm{C}-\mathrm{X}_{4}-\mathrm{C}-\mathrm{X}_{13}-\mathrm{C}-\mathrm{X}_{2}-\mathrm{C}\right)$ [35]. Regarding the human sequence, the $\mathrm{C}$-terminal region in Leishmania RPA-1 exhibits amino acid sequence differences, especially on the last 54 amino acids (Figure 1). Given the role of the C-terminal end for the trimerization of the RPA complex [36], and the fact that RPA protein has been considered as a target for cancer drug development [3,11], the
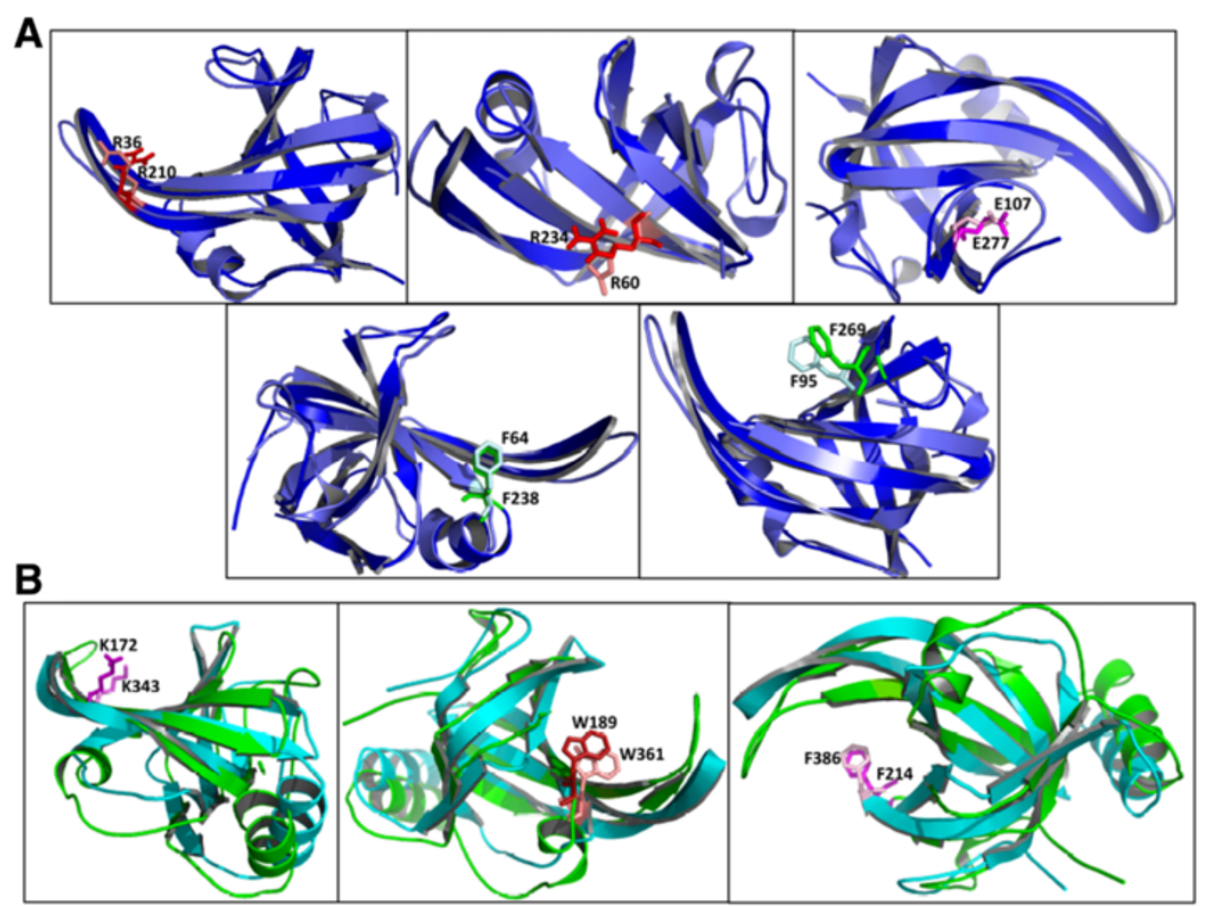

Figure 3 Superposition of structures and relevant amino acids between LbRPA-1 and hRPA-1 3D models in the DBD-A (panels A) and DBD-B (panels B) domains. In panels $\mathbf{A}$, light coloured structures correspond to LbRPA-1 and dark coloured ones to hRPA-1. In panels $\mathbf{B}$, light colors were used for hRPA-1 and dark colors for LbRPA-1. 
differences at this region might be exploited for the discovery of molecules with pharmacological potential that perturb the complex formation of the RPA protein, following a similar approach to that used for the triosephosphate isomerase of T. cruzi [37].

RPA-1 undergoes phosphorylation, which is believed to play a role in modulating its affinity for DNA. In humans, the Chk1 protein, a serine/threonine kinase, phosphorylates with high efficiency the DBD-A and DBD-B domains (171-450 amino acids) [38]. As expected, it was noted that phosphorylation decreases the affinity of these two major DNA-binding domains [38]. Moreover, binding of RPA-1 to ssDNA blocks Chk1 phosphorylation, suggesting that Chk1 and ssDNA compete for binding to the RPA domains [38]. In this regard, the analysis of phosphorylation sites in L. braziliensis and L. amazonensis RPA-1 proteins showed the presence of at least 25 potential sites, sixteen of which are located on the DBD-A and DBD-B domains (Figure 1).

Molecular modeling of the L. braziliensis RPA-1 protein was carried out, firstly, using as template the $U$. maydis RPA-1 sequence (PDB identification number 4 GOP). Both proteins share $34 \%$ of sequence identity (Figure 1). For crystallization purpose, the RPA-1 subunit of the fungus $U$. maydis was truncated to remove the N-terminal OB fold [19]. Nevertheless, as the Leishmania RPA-1 naturally lacks of this N-terminal domain

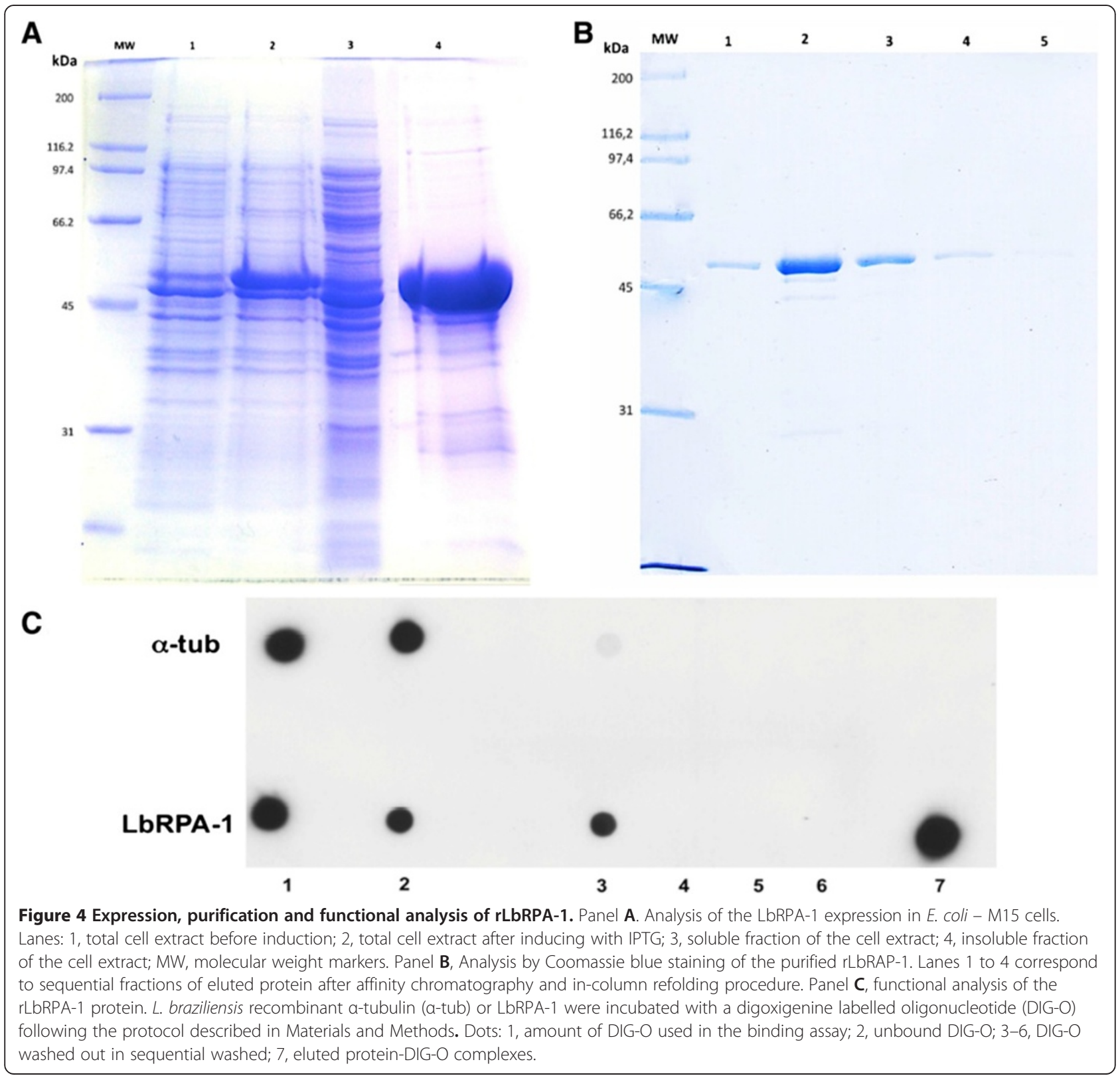


(see above), most of LbRPA-1 sequence aligns with the resolved structure of $U$. maydis RPA- 1 . The truncated $U$. maydis RPA was crystallized bound to oligodeoxythymide ssDNA of either $62 \mathrm{nt}$ or $32 \mathrm{nt}$, and the structures were refined at $2.8-\mathrm{A}^{\circ}$ and $3.1-\mathrm{A}^{\circ}$ resolution, respectively [19]. Interestingly, a tridimensional model covering from position 9 to 455 of LbRPA-1 was obtained using the Phyre2 program (Figure 2A). This model has been deposited in the Protein Model database (PMBD) with the ID PM0079361. The Ramachandran plot statistics showed that $81.4 \%$ of the residues in the modelled LbRPA-1 are in the most allowed regions, $15.9 \%$ in additional allowed regions, $2 \%$ in generously allowed regions and only $0.8 \%$ in disallowed regions. These data indicate that the Leishmania modelled protein has a quality similar to that determined for the $U$. maydis X-ray resolved structure. Thus, the Ramachandran plot data indicated that the build model is appropriate and suitable for further analyses. Additionally, the LbRPA-1 sequence was submitted to the iterative threading assembly refinement (I-TASSER) server, which carry out a fully automated protein prediction based on iterative structural assemblies with tridimensional models of known proteins. The more probable structure generated by this method (Figure $2 \mathrm{~B}$ ) resulted to be very similar to that generated by Phyre2 program (Figure 2A). Unsurprisingly, the I-TASSER server also found the $U$. maydis RPA-1 as the more adequate model for modelling of LbRPA-1. In panel C of Figure 2, it is shown the structural alignment of both protein models (LbRPA-1 and UmRPA-1) that was generated using the MacPyMOL program. A root mean-square deviation (RMSD) value of $0.109 \AA$ was obtained; this value suggests that, in spite of the genetic distance between these two types of organisms, the overall structure of both RPA-1 is maintained.

In the human RPA-1 (hRPA-1) several amino acids have been identified as crucial for DNA binding [7], we analyze if they were also present in equivalent structural positions in the LbRPA-1. Remarkably, R210, R234, F238, F269 and E277 residues in the DBD-A domain of hRPA- 1 were found conserved in the DBD-A domain of LbRPA-1; they corresponds to the residues R36, R60, F64, F95 and E103, respectively (Figure 3A). Similarly, the K172, W189 and F214 amino acids of the LbRPA-1 protein would be the equivalent to residues K343, W361 and F386 described as crucial for DNA-binding in the DBD-B domain of hRPA-1 (Figure 3B). In all, these findings pointed to a functional conservation of the DNAbinding capacity between hRPA-1 and the L. braziliensis LbRPA-1 protein.

\section{Expression of L. braziliensis RPA-1 as recombinant protein} and analysis of its affinity to ssDNA

For expression of recombinant L. braziliensis RPA-1, oligonucleotides were designed from the GeneDB entry LbrM.28.1990 (see Methods for further details), and the coding region was cloned into the $E$. coli pQE30 expression vector. The expression of the protein was observed after IPTG addition to the culture medium (Figure 4A). However, subcellular fractionation evidenced that most of the protein was forming insoluble polypeptide aggregates, i.e. inclusion bodies. A similar finding was observed by Lira and co-workers when expressing the $L$. amazonensis RPA-1 in E. coli [25]. In order to solubilize
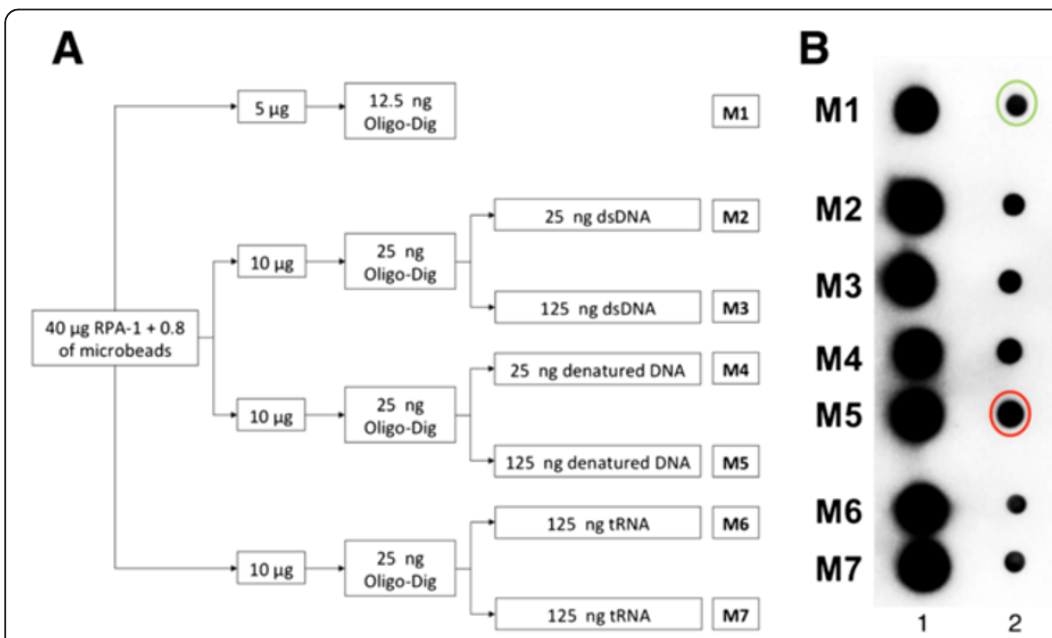

Figure 5 Qualitative analysis of the affinity of rLbRPA-1 for ssDNA. Panel A, scheme showing the experimental design. Panel B, follow up of the digoxigenin-labelled oligonucleotide (Oligo-Dig) in the different steps of the pull down assay. For each experiment, the analyzed samples were: 1, total amount of oligo-DIG used in the assay (12.5 ng); 2, unbound oligo-DIG after incubation with rLbRAP-1 containing beads; 3-5, Oligo-DIG presents in sequential washes; 6 and 7, 1/4 and 1/16 dilutions, respectively, of the eluted rLbRPA-1-oligo-DIG complexes. Green circles in samples two and six from mixture 1 (M1) indicate, respectively, unbound (2) and 1/4 of the eluted oligonucleotide (6), as signal intensity control, whilst red circles mark equivalent samples of the mixture 5 (M5) in which the oligo-DIG binding was compited by ssDNA. 
the protein, these authors developed a purification protocol that allowed to recover the protein in a highly pure and active form. Therefore, we followed that procedure with minimal modifications (see Methods), and finally it was possible to obtain the L. braziliensis rRPA-1 in a soluble form (Figure 4B).

After purification of the rLbRPA-1, we analyzed whether the protein maintained its cognate capacity of binding to ssDNA. For this purpose, an oligodeoxyribonucleotide, containing a digoxigenin molecule at its $5^{\prime}$-end (DIG-O), was synthetized and used in binding assays with the recombinant protein. The ability of the rLbRPA-1 to bind this DIG-O was tested by pull-down assays based on the affinity of the His-tag to $\mathrm{Ni}^{2+}$-beads. As shown in Figure 4C, rLbRPA-1 showed a clear affinity for ssDNA, suggesting that the protein was refolded in vitro correctly. As a negative control, binding assays using recombinant L. braziliensis $\alpha$-tubulin were performed in parallel.

To further characterize the substrate specificity of rLbRPA-1, competition assays using dsDNA, ssDNA or tRNA were carried out (Figure 5). After binding of rLbRPA-1 to the DIG-O, only a five molar excess of ssDNA was able to significant displace the bound DIGO. No competition was observed when dsDNA was

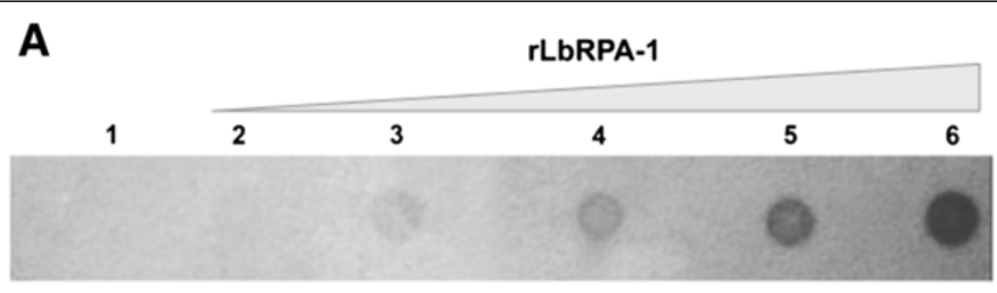

B

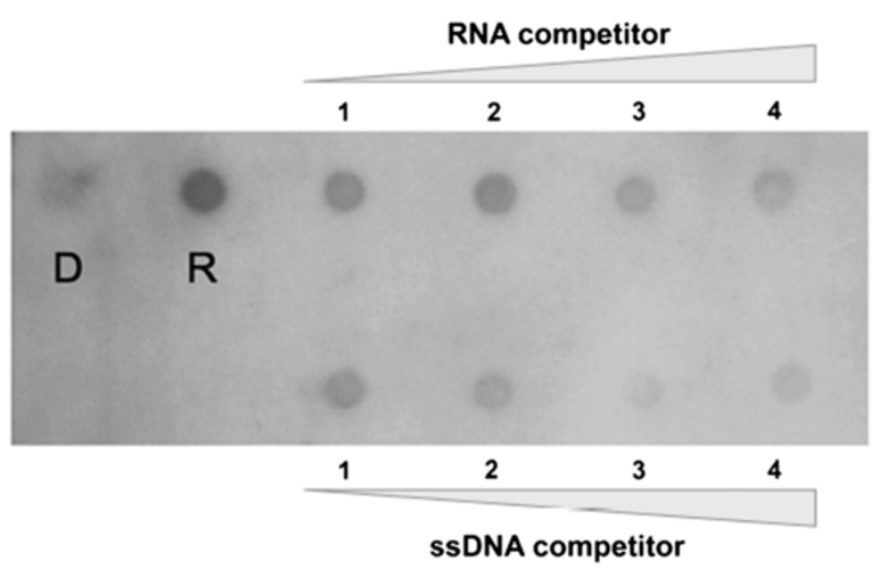

C

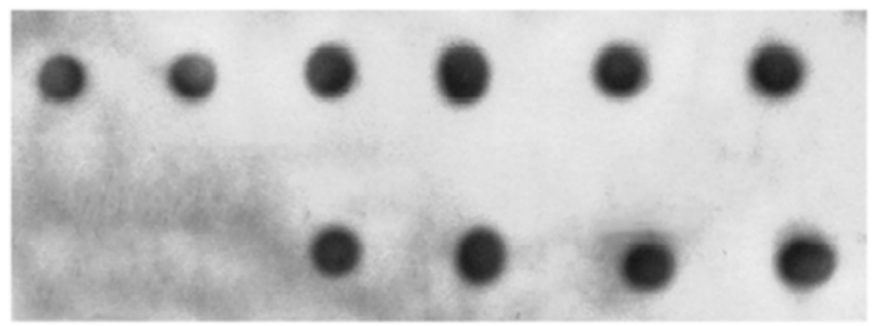

Anti-His antibody

Figure 6 Analysis of the RNA-binding capacity of the rLbRPA-1 protein and its relative affinity for RNA and ssDNA. (Panel A) Two hundred ng of digoxigenin labeled 3' HSP70 UTR-II were incubated with beads containing different amounts of rLbRPA-1: 0 (dot 1), 0.05 pM (2), $0.25 \mathrm{pM}$ (3), $1.25 \mathrm{pM}(4), 6.25 \mathrm{pM}(5)$ and $31.25 \mathrm{pM}$ (6). After whasing out of the unbound RNA, eluted complexes were deposited on the membrane and the remaining bound RNA was monitored by an anti-digoxigenin antibody. (Panel B) One pM of either denatured (dot D) or renatured rLbRPA-1 (dot R) was incubated with the digoxigenin-labeled RNA oligonucleotide (100 fM). Additionally, the binding of the Dig-RNA oligonucleotide to the renatured rLbRPA-1 was competited with increasing amounts of either the non-labelled RNA oligonucleotide (upper array) or a non-labelled DNA oligonucleotide (lower array) containing the equivalent sequence: $25 \mathrm{fM}$ (dot 1), $50 \mathrm{fM}$ (2), $100 \mathrm{fM}$ (3) and $200 \mathrm{fM}$ (4). After whasing out the unbound oligonucleotides, the complexes were eluted and put on the membrane, which was revealed by an anti-digoxigenine antibody. (Panel $\mathbf{C}$ ) The membrane of panel B was incubated with an anti-his-tag antibody in order to monitor that equivalent amounts of rLbRPA-1 was present in the different samples. 
used in excess. Also, no displacement of the DIG-O was attained after incubation with yeast tRNA (Figure 5). In summary, from these experiments, it must be concluded that rLbRPA-1, as described for homologous proteins in other organisms, is essentially a ssDNA binding protein.

\section{L. braziliensis RPA-1 also binds RNA}

Although the rLbRPA-1 showed to have a clear affinity for ssDNA, we addressed experimentally whether the protein can also interact with RNA. It should be noted that this protein was initially identified by its binding to the untranslated regions of the HSP70 mRNAs [18]. On the other hand, Cano and co-workers described that $L$. amazonensis RPA-1 was able to interact with an RNA oligonucleotide containing the Tetrahymena telomeric sequence [15]. Moreover, there are many proteins of the T. brucei editosome, involved in RNA editing, that contains the OB-fold domain [39]. In order to study an rLbRPA-1/RNA interaction, we designed non-competitive and competitive pull-down assays. For this purpose, after purifying the recombinant LbRPA-1, it was bound to $\mathrm{Ni}^{2+}$-beads and incubated with digoxigenin-labeled RNA containing the 3'UTR of L. braziliensis HSP70-II gene. Interestingly, it was observed that rLbRPA-1 interacts with RNA and the amount of bound RNA increased in parallel with the amount of protein present in the sample (Figure 6A). These results indicated that rLbRPA-1 has also RNA-binding activity.

Once the RNA binding activity of rLbRPA-1 was demonstrated, we considered of interest to further evaluate its relative affinity for DNA and RNA. For this purpose, a digoxigenin-labelled RNA oligonucleotide (derived from the 3' HSP70-II UTR RNA sequence; 5' 5DigNAUGUCUUUUA UUUUUUUGUG UGUGUUUUAU AUUUUUCUCC UUUCGUACUA A 3') was incubated with the renatured rLbRPA-1 and its binding analyze also by pull-down assays (Figure 6B). In this assay, we included an assay in which the rLbRPA-1 was maintained in a denatured conformation by adding $8 \mathrm{M}$ urea to the binding buffer. This control served to exclude that the oligonucleotide binding was non-specific (Figure 6B, point D). Additionally, competition assays using either the non-labelled RNA oligonucleotide or a DNA oligonucleotide containing the equivalent sequence ( 5 ' ATGT CTTTTA TTTTTTTGTG TGTGTTTTAT ATTTTTC TCC TTTCGTACTA A 3') were carried out. The results showed that the competition was more effective in the presence of the DNA oligonucleotide competitor than with the RNA one. These findings confirmed the higher affinity of rLbRPA-1 for ss-DNA than for RNA, at least when this particular sequence, present in the 3' HSP70-II mRNA molecule, is used as substrate. Nevertheless, for future works, it should be analyzed the relative affinity for DNA and/or RNA of the native LbRPA-1, considering that heterologous expression of rLbRPA-1 (as for any other protein) in $E$. coli could have introduced conformational alterations or modifications that may affect in some extent the affinity for its targets.

\section{Conclusions}

The rLbRPA-1 protein, purified in denaturing conditions and refolded in vitro, was able to bind ssDNA molecules. This result confirmed, on one hand, that the purified protein is functional and, on the other hand, allowed us to confirm that LbRPA-1 is a ssDNA binding protein as predicted. In addition, we have shown that this protein is able to interact with RNA molecules, which may be suggesting a role of this protein in the mRNA HSP70 expression, in particular, and in RNA metabolism, in general. Likewise, the detailed structural analysis and the molecular modelling carried out on the Leishmania RPA-1 protein have shown a remarkable conservation with the homologues from evolutionarily distant organisms. Nevertheless, the comparison with the human RPA-1 protein also served to locate differences, such as the absence of the N-terminal RPA70N domain and the existence of a highly divergent sequence at the $\mathrm{C}$ - terminal region. These differences could be exploited for developing molecules with antileishmanial properties.

\section{Competing interests}

The authors declare that they have no competing interests.

\section{Authors' contributions}

PANM, CAR, JMR, and CJP conceived and designed the experiments. GEB and JG advised the bioinformatics analyses. PANM and CAR performed the analyses and experiments. PANM, CAR, JMR, and CJP analyzed the data and wrote the paper. All authors read and approved the final version of the manuscript.

\section{Acknowledgments}

This work was supported by Pontificia Universidad Javeriana (Colombia) Research Project ID PPTA 00004564 "Estudio de la interacción entre la proteína Rpa1 y la región 3 UTR-II de los genes HSP70 de Leishmania braziliensis". PANM and CAR were supported by Colciencias, Programa Nacional de Doctorados 2012, and 2008, respectively. Work at the JMR's lab was supported by VI PN de I + D + I 2008-2011, ISCIII -Subdirección General de Redes y Centros de Investigación Cooperativa (RICET- RD12-0018-0009). JMR's ORCID ID: 0000-00033410-9488.

\section{Author details}

${ }^{1}$ Laboratorio de Parasitología Molecular, Facultad de Ciencias, Pontificia Universidad Javeriana, Carrera 7 No 43-82, Edificio 50, Laboratorio 113 Bogotá, Colombia. ${ }^{2}$ Departamento de Nutrición y Bioquímica, Facultad de Ciencias, Pontificia Universidad Javeriana, Bogotá, Colombia. ${ }^{3}$ Centro de Biología Molecular Severo Ochoa (CSIC-UAM), Universidad Autónoma de Madrid, Madrid, Spain.

Received: 17 April 2014 Accepted: 26 November 2014

Published online: 12 December 2014

\section{References}

1. Alvar J, Velez ID, Bern C, Herrero M, Desjeux P, Cano J, Jannin J, den Boer M: Leishmaniasis worldwide and global estimates of its incidence. PLOS ONE 2012, 7:e35671.

2. Velez ID, Hendrickx E, Robledo SM, Agudelo SP: Leishmaniosis cutánea en Colombia y género. Cad Saúde Pública 2001, 17:171-180. 
3. Zou Y, LiU Y, Wu X, Shell SM: Functions of human replication protein A (RPA): From DNA replication to DNA damage and stress responses. J Cell Physiol 2006, 208:267-273.

4. Lindsey-Boltz LA, Reardon JT, Wold MS, Sancar A: In vitro analysis of the role of replication protein A (RPA) and RPA phosphorylation in ATR-mediated checkpoint signaling. J Biol Chem 2012, 287:36123-36131.

5. Wold MS: Replication protein A: a heterotrimeric, ssDNA-binding protein required for eukaryotic DNA metabolism. Annu Rev Biochem 1997, 66:61-91.

6. Bochkarev A, Bochkareva E: From RPA to BRCA2: lessons from singlestranded DNA binding by the OB-fold. Curr Opin Struct Biol 2004, 14:36-42.

7. Bochkarev A, Pfuetzner RA, Edwards AM, Frappier L: Structure of the singlestranded-DNA-binding domain of replication protein A bound to DNA. Nature 1997, 385:176-181.

8. Jacobs DM, Lipton AS, Isern NG, Daughdrill GW, Lowry DF, Gomes X, Wold MS: Human replication protein A: Global fold of the N-terminal RPA-70 domain reveals a basic cleft and flexible C-terminal linker. J Biomol NMR 1999, 14:321-331.

9. Fanning $E$, Klimovich $V$, Nager AR: A dynamic model for replication protein A (RPA) function in DNA processing pathways. Nucleic Acids Res 2006, 34:4126-4137.

10. Lao Y, Gomes XV, Ren Y, Taylor JS, Wold MS: Replication protein A interactions with DNA. III Molecular basis of recognition of damaged DNA. Biochemistry 2000, 39:850-859.

11. Patrone JD, Kennedy JP, Frank AO, Feldkamp MD, Vangamudi B, Pelz NF, Rossanese OW, Waterson AG, Chazin WJ, Fesik SW: Discovery of proteinprotein interaction inhibitors of replication protein A. ACS Med Chem Lett 2013, 4:601-605.

12. Brown GW, Melendy TE, Ray DS: Conservation of structure and function of DNA replication protein $A$ in the trypanosomatid Crithidia fasciculata. Proc Natl Acad Sci 1992, 89:135-142.

13. Brown GW, Hines JC, Fisher P, Ray DS: Isolation of the genes encoding the 51-kilodalton and 28-kilodalton subunits of Crithidia fasciculata replication protein A. Mol Biochem Parasitol 1994, 63:135-142.

14. Barral A, Pedral-Sampai D, Grimaldi G Jr, Momen H, McMahon-Pratt D, Ribeiro de Jesus A, Almeida R, Badaro R, Barral-Netto M, Carvalho EM, Jhonson WD Jr: Leishmaniasis in Bahia, Brazil: evidence that Leishmania amazonensis produces a wide spectrum of clinical disease. Am J Trop Med Hyg 1991, 44:536-546

15. Fernández MF, Castellari RR, Conte FF, Gozzo F, Sabino AA, Pinheiro H, Novello JC, Eberlin MN, Cano MI: Identification of three proteins that associate in vitro with the Leishmania (Leishmania) amazonensis G-rich telomeric strand. Eur J Biochem 2004, 271:3050-3063.

16. Siqueira-Neto JL, Lira CBB, Giardini MA, Khater L, Perez AM, Peroni LA, dos Reis JR, Freitas-Junior LH, Ramos CH, Cano Ml: Leishmania replication protein A-1 binds in vivo single-stranded telomeric DNA. Biochem Biophys Res Commun 2007, 358:417-423.

17. Viveiros Da Silveira RC, Santos Da Silva M, Nunes VS, Perez AM, Cano Ml: The natural absence of RPA1N domain did not impair Leishmania amazonensis RPA-1 participation in DNA damage response and telomere protection. Parasitology 2013, 140:547-559.

18. Ramírez CA, Dea-Ayuela MA, Gutiérrez-Blázquez MD, Bolas-Fernández F, Requena JM, Puerta CJ: Identification of proteins interacting with HSP70 mRNAs in Leishmania braziliensis. J Proteomics 2013, 94:124-137.

19. Fan J, Pavletich NP: Structure and conformational change of a replication protein A heterotrimer bound to ssDNA. Genes Dev 2012, 26:2337-2347.

20. Ramírez CA, Requena JM, Puerta CJ: Identification of the HSP70-II gene in Leishmania braziliensis HSP70 locus: genomic organization and UTRs characterization. Parasit Vectors 2011, 4:166-177

21. Notredame C, Higgins DG, Heringa J: T-coffee: a novel method for fast accurate multiple sequence alignment. J Mol Biol 2000, 302(1):205-217.

22. Zdobnov EM, Apweiler R: InterProScan- an integration platform for the signature-recognition methods in InterPro. Bioinformatics 2001, 17:847-848.

23. Quevillon E, Silventoinen V, Pillai S, Harte N, Mulder N, Apweiler R, Lopez R: InterProScan: protein domains identifier. Nucleic Acids Res 2005, 33:W116-W120

24. Blom N, Gammeltoft S, Brunak S: Sequence and structure-based prediction of eukaryotic protein phosphorylation sites. J Mol Biol 1999, 294(5):1351-1362

25. Lira CB, Gui KE, Perez AM, da Silveira RC, Gava LM, Ramos CH, Cano MI: DNA and heparin chaperone the refolding of purified recombinant replication protein A subunit 1 from Leishmania amazonensis. Biochim Biophys Acta 2009, 1790(2):119-125.

26. Altschul SF, Madden TL, Schäffer AA, Zhang J, Zhang Z, Miller W, Lipman DJ: Gapped BLAST and PSI-BLAST: a new generation of protein database search programs. Nucleic Acids Res 1997, 25:3389-3402

27. Westbrook J, Feng Z, Chen L, Yang H, Berman HM: The Protein Data Bank and structural genomics. Nucleic Acids Res 2003, 31:481-491.

28. Kelley LA, Sternberg MJ: Protein structure prediction on the Web: a case study using the Phyre server. Nat Protoc 2009, 4:363-371.

29. Laskowski RA, MacArthur MW, Moss DS, Thornton JM: PROCHECK: a program to check the stereochemical quality of protein structures. J App/ Crystallogr 1993, 26:283-291.

30. Arnold K, Bordoli L, Kopp J, Schwede T: The SWISS-MODEL Workspace: a web-based environment for protein structure homology modelling. Bioinformatics 2006, 22:195-201.

31. Zhang Y: I-TASSER server for protein 3D structure prediction. BMC Bioinformatics 2008, 9:40.

32. Roy A, Kucukural A, Zhang Y: I-TASSER: a unified platform for automated protein structure and function prediction. Nat Protoc 2010, 5:725-738.

33. Roy A, Yang J, Zhang Y: COFACTOR: an accurate comparative algorithm for structure-based protein function annotation. Nucleic Acids Res 2012, 40:W471-W477.

34. Erdile LF, Heyer WD, Kolodner R, Kelly TJ: Characterization of a cDNA encoding the 70-kDa single-stranded DNA-binding subunit of human replication protein $A$ and the role of the protein in DNA replication. J Biol Chem 1991, 266:1209-12098.

35. Heyer WD, Rao MRS, Erdile LF, Kelly TJ, Kolodner RD: An essential Saccharomyces cerevisiae single-stranded DNA binding protein is homologous for the large subunit of human RP-A. EMBO J 1990, 9:2321-2329.

36. Bochkareva E, Koroley S, Lees-Miller SP, Bochkarev A: Structure of the RPA trimerization core and its role in the multistep DNA-binding mechanism of RPA. EMBO J 2002, 21:1855-1863.

37. Olivares-Illana V, Rodríguez-Romero A, Becker I, Berzunza M, García J, PérezMontfort R, Cabrera N, López-Calahorra F, de Gómez-Puyou MT, GómezPoyou A: Perturbation of the dimer interface of triosephosphate isomerase and its effect on Trypanosoma cruzi. PLoS Negl Trop Dis 2007, 1:e1.

38. Liu JS, Kuo SR, Melendy T: DNA damage-induced RPA focalization is independent of gamma-H2AX and RPA hyper-phosphorylation. J Cell Biochem 2006, 99:1452-1462.

39. Göringer HU: 'Gestalt', composition and function of the Trypanosoma brucei editosome. Annu Rev Microbiol 2012, 66:65-82.

\section{doi:10.1186/s13071-014-0573-8}

Cite this article as: Nocua et al:: Leishmania braziliensis replication protein A subunit 1: molecular modelling, protein expression and analysis of its affinity for both DNA and RNA. Parasites \& Vectors 2014 7:573.

\section{Submit your next manuscript to BioMed Central and take full advantage of:}

- Convenient online submission

- Thorough peer review

- No space constraints or color figure charges

- Immediate publication on acceptance

- Inclusion in PubMed, CAS, Scopus and Google Scholar

- Research which is freely available for redistribution 